\title{
DIAGNOSTIC, AND THERAPEUTIC CHALLENGES IN HIV+ PATIENT, PRESENTED WITH LEFT-SIDED HAEMIPARESIS AND MRI CHANGES
}

\author{
Vanya Georgieva ${ }^{1}$, Antonina Dineva ${ }^{1}$, Vakril Nikolov ${ }^{1}$, Mariana Stoycheva $^{1}$, Borislav Milev $^{2}$ \\ ${ }^{1}$ St. George University Hospital, Department of Infectious Diseases Parasitology and \\ Tropical Medicine, Plovdiv, Bulgaria \\ ${ }^{2}$ Medical Diagnostic Laboratory - Rusev
}

\begin{abstract}
Neurological features occur during the later stages of HIV infection. The most common manifestations are opportunistic infections like cerebral toxoplasmosis and progressive multifocal leukoencephalopathy, as well as malignant lymphomas like primary EBV associated CNS lymphoma. We present a case of a 21-yearold man, diagnosed as HIV+ in 2010. The patient receives HAART (kivexa/kaletra) from 13.08.2013 to date with poor adherence. The current complaints began two weeks ago with weakness in the lower extremities, gait disturbances and headache. The neurological examination at the admission time showed no meningeal signs, weakened tendon reflexes in left limbs, absent abdominal reflexes, central facial palsy on the left, positive Babinski sign. Upon admission to the hospital the patient presented with CD $4-34 \mathrm{c} / \mathrm{mm}^{3}, \mathrm{VL}-2180$ $\mathrm{c} / \mathrm{ml}$. Cerebral spinal fluid viral load was $<20 \mathrm{c} / \mathrm{ml}$. The results of the serological and virological tests from cerebral spinal fluid were: PCR EBV (-); PCR CMV (-); IgM EBV (-); IgG CMV (-); T.gondii IgM (-); IgG(-); PCR for pathogenic free-living amoeba (-). MRI: space occupying lesion, interpreted as a lymphoma, MRI after 6 months: progression of the lesion. A biopsy of peripheral lymph node showed nonspecific finding. The patient was put on patogenetic treatment against cerebral edema, treatment against $T$. gondii infection (clindamycin, co-trimoxazole), and gancyclovir, meronem, fungolon, ART as well. The patient was without precise, laboratory confirmed diagnosis, but the general condition is better.
\end{abstract}

Keywords: HIV, hemiparesis, MRI

\section{INTRODUCTION}

Most of the neurological manifestations of the HIV infection occur in the later stages with the progression of the immunodeficiency, even though the

Address for correspondence:

Vanya Georgieva

Department of Infectious Diseases Parasitology

and Tropical Medicine

St. George University Hospital

66 Peshtersko Shose blvd.

4002 Plovdiv, Bulgaria

e-mail:vania.b.georgieva@gmail.com

Received: October 29, 2014

Accepted: March 11, 2015 central nervous system can be involved in every stage of the HIV infection.

The most common neurological opportunistic infection is cerebral toxoplasmosis (4). Clinical symptoms depend on the localization of lesions. The major signs include focal neurological deficit: paresis, speech problems or sensory loss, seizures (8). Headache with fever or subfebrile temperatures are always suspicious. Meningitis signs, however, are less typical. The diagnosis is made by performing a MRI. An updated serology should be available for every patient. Up to $97 \%$ of patients with cerebral toxoplasmosis have IgG+ antibodies, and so a negative result makes toxoplasmosis unlikely. IgM is only rarely positive (4). 
Vanya Georgieva, Antonina Dineva, Vakril Nikolov et al.

Similar clinical features in a patient with severe immunodeficiency can cause JCV virus infection, which results in progressive multifocal leukoencephalopathy (PML) (6). The main focus of the disease is the white matter of the cerebral hemispheres, but in some cases, the cerebellum, the grey matter may also be affected. The clinical signs of the disease include cognitive disorders, which may range from mild impairment of concentration to dementia, focal neurological deficits, mono- and hemiparesis, speech and visual deficits, seizures. The best method for establishing the diagnosis is MRI, which also allows clarification between cerebral toxoplasmosis and lymphoma. Lesions are often parieto-occipital or periventricular, but the cerebellum may also be involved. Examination of cerebrospinal fluid is essential. CSF should always be tested for JCV, but the negative PCR best method in establishing the diagnosis is MRS, which presents a variety of biochemical changes: lactate/lipid peak, increased choline/creatine and reduced $\mathrm{N}$-acetylaspartate/creatine ratios in the lesion $(5,7)$. Quantitative EBV PCR in the CSF improves the diagnostic specificity, although the predictive value remains too low for it to be used as an isolated marker for PCNSL (2).

The differential diagnosis includes cryptococcosis, glioblastoma, metastatic lesions.

\section{CASE PRESENTATION}

A 21-year-old man, intravenous drug user on methadone treatment, diagnosed as HIV+ in 2010. He had been started on kaletra/viread/emtriva at the time of diagnosis, but soon after that was lost to follow-up. In 2013 the patient was started on kivexa/ kaletra (Tabl. 1).

Tabl. 1. Tests results

\begin{tabular}{lc|c|c}
\hline Date & CD 4 & VL & HAART \\
\hline $\mathbf{0 8 . 0 9 . 2 0 1 0}$ & 41 & 15200 & Kaletra/viread/emtriva \\
$\mathbf{1 7 . 1 1 . 2 0 1 0}$ & 15 & 215000 & 14700 \\
$\mathbf{1 6 . 0 3 . 2 0 1 1}$ & 17 & 75800 & $\rightarrow$ Kivexa/Kaletra \\
$\mathbf{2 7 . 0 7 . 2 0 1 1}$ & 13 & - & 2180 \\
$\mathbf{1 9 . 0 6 . 2 0 1 3}$ & 6 & 1860 & \\
$\mathbf{1 3 . 0 8 . 2 0 1 3}$ & 34 & 68 & \\
$\mathbf{0 8 . 0 1 . 2 0 1 4}$ & &
\end{tabular}

does not exclude the diagnosis $(1,3)$.

The differential diagnosis include also primary CNS lymphomas (PCNSL), associated in almost $100 \%$ of the cases with EBV (2). Histological findings are almost always consistent with diffuse large cell non-Hodgkin's lymphomas (7). Different neurological deficits occur depending on the localization. Epileptic seizures, personality changes, changes in awareness, headache and focal deficits such as paresis are frequent. For establishing the diagnosis is necessary to be performed an emergency CT or (better) MRI. The most important differential diagnosis is cerebral toxoplasmosis. A solitary mass is usually more indicative of primary CNS lymphomas, however 2-4 lesions may be present. More than four lesions of a primary CNS lymphomas are rarely found. The
The current complaints began two weeks ago with weakness in the lower extremities, gait disturbances, headache, weight loss. At the time of admission the patient was afebrile, with peripheral lymphadenomegaly- swollen lymph nodes in the cervical area - beneath the sternocleidomastoid muscles, and in the right axillary area. The nodes were painless, dense, with size $1 / 1 \mathrm{sm}$. There were no abnormalities in the respiratory and cardiovascular systems. The neurological examination showed absent meningeal signs, weakened deep tendon reflexes for left extremities, absent abdominal reflexes, central facial palsy on the left, positive Babinski sign.

The laboratory tests showed mild anaemia and hypoproteinemia. The patient's viral load at the admission was $2180 \mathrm{c} / \mathrm{ml}, \mathrm{CD} 4-34 \mathrm{c} / \mathrm{mm}^{3}$. A lumbar 
Tabl. 2. CSF results

\begin{tabular}{ll} 
WBC & $6 / \mu \mathrm{l}$ \\
CSF-protein & $0,6 \mathrm{~g} / \mathrm{l}$ \\
CSF-glucose & $2,9 \mathrm{mmol} / \mathrm{l}$ \\
VL & $<20 \mathrm{c} / \mathrm{ml}$ \\
EBV PCR & $(-)$ negative \\
CMV PCR & $(-)$ negative \\
EBV IgM, IgG & $(-)$ negative \\
CMV IgM, IgG & $(-)$ negative \\
T. gondi IgM, IgG & $(-)$ negative \\
PCR for free-living amoeba & $(-)$ negative \\
Culture of free-living amoeba & $(-)$ negative \\
\hline \hline
\end{tabular}

puncture was performed - with no abnormalities (Tabl. 2). The pulmography and abdominal ultrasound were also normal. The patient was consulted with a haematologist. As a result lymph node biopsy was performed without evidence of tumor cells. A MRI with MRS was performed. The results: different lesions in the brain parenchyma, the most distinct is hypointense lesion in right parietal lobe with high signal intensity on T1 and T2. There was no evidence of cortical atrophy, CSF spaces had normal placement and capacity. The conclusion was: lymphoma. The spectroscopy of the patient was also interpreted as lymphoma: significant elevation in choline/creatine ratio and $\mathrm{N}$-acetyl aspartate depression (Fig. 1, 2, 3, 4).

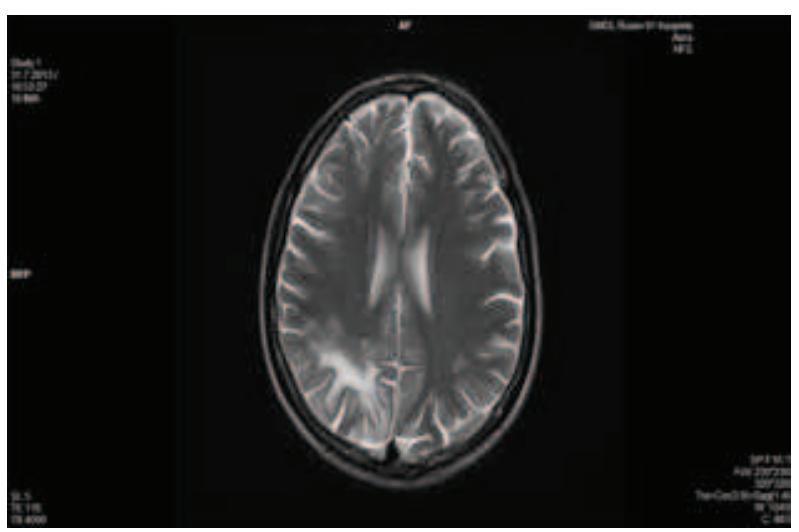

Fig. 1. MRI at time of diagnosis: different lesions in the brain parenchyma, the most distinct is hypointense lesion in right parietal lobe with high signal intensity on $\mathrm{T} 1$ and $\mathrm{T} 2$

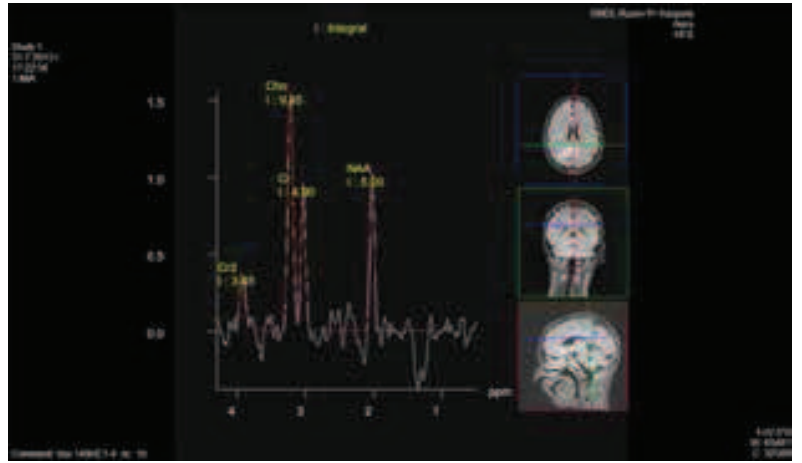

Fig. 2. MRS-CSI- chemical shift 3D at time of diagnosis: significant elevation in choline/creatine ratio and $N$-acetyl aspartate depression

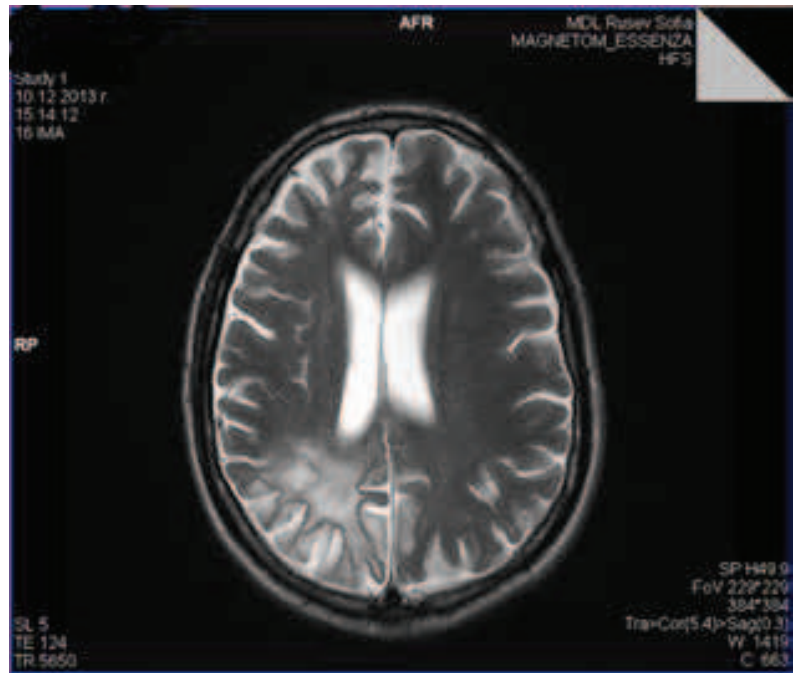

Fig. 3. MRI- second: on the control MRI scan the known from the first MRI scan lesion in the right parietal lobe has been enlarged, there is also presence of focal atrophy of the frontal and parietal lobes

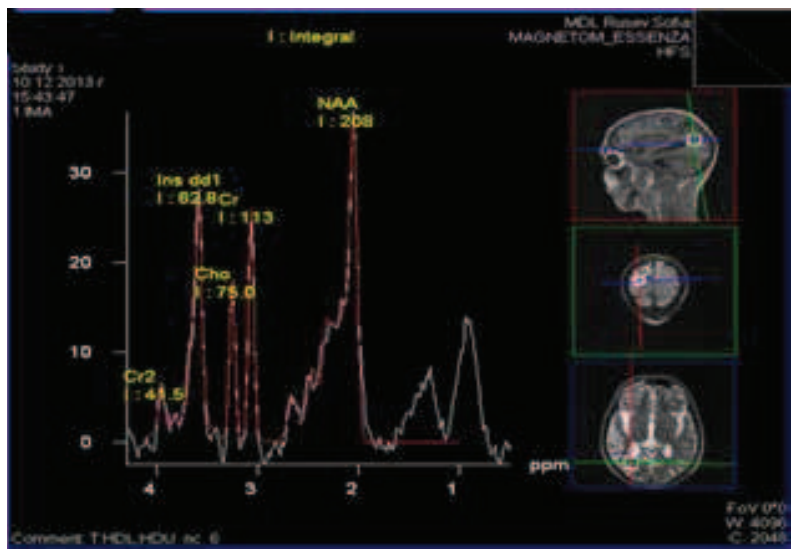

Fig. 4. MRS-CSI- chemical shift 3D: second scan. Compared to the first MRS here we see improvement of the neuronal function. The choline rate is reduced, $N$-acetylaspartatel creatine ratio is higher than choline/creatine ratio 
Vanya Georgieva, Antonina Dineva, Vakril Nikolov et al.

Since admission the patient was put on intravenous rehydration. He underwent a course of mannitol treatment, as well as human serum albumin, without any result. Because of the suspicion of cerebral toxoplasmosis we started treatment with clindamycin and co-trimoxazole. He received also a course with ganciclovir, meronem and fungolon. In the course of treatment there was an improvement of the clinical condition of the patient, and after 6 months there was almost a complete recovery of the neurological findings. The second MRI was performed 6 months after the beginning of the illness and showed enlargement of the lesion in the right parietal lobe, with presence of focal atrophy in the frontal and parietal lobes.

\section{DISCUSSION}

The clinical case presents a HIV+ patient with left hemiparesis and lesion in the right parietal lobe. There were numerous difficulties in establishing the diagnosis. The HIV+ status of the patient, as well as the poor adherence to HAART were leading to severe immunodeficiency, which was the reason for a reduced immune response. Primary CNS lymphoma and cerebral toxoplasmosis are the leading causes of focal brain lesions in HIV+ patients. For that reason we performed a MRI. That brought us to the diagnosis of lymphoma, which we were not able to confirm. Unfortunately, the patient was not indicated for brain biopsy. In regard to toxoplasmosis, all serological and virological tests were negative, which also did not exclude the diagnosis. There was clinical improvement as a result of the toxoplasmosis treatment, but the MRI showed progression of the disease. We could not also confirm some rare possibilities as CMV encephalitis and free-living amoeba encephalitis.

The patient was subjected to a follow up, although for now he remains without a laboratory confirmed diagnosis.

\section{CONCLUSIONS}

Establishing a diagnosis of toxoplasmosis is not easy, especially in HIV+ patients, because of their severe immunodeficiency. In this case an improvement was detected after initiating antitoxoplasmosis treatment. Further, we accept cerebral toxoplasmosis as a diagnosis, because of the clinical improvement.

\section{REFERENCES}

1. Bossolasco S, Calori G, Moretti F, et al. Prognostic significance of JC virus DNA levels in cerebrospinal fluid of patients with HIV- associated progressive multifocal leukoencephalopathy. Clin Infect Dis, 2005;40:738-44.

2. Camillieri-Broet S, Davi F, Feuillard J, et al. AIDSrelated primary brain lymphomas: histopathologic and immunohistochemical study of 51 cases. Hum Pathol 1997;28:367-74.

3. Garcia De Viedma D, Diaz Infantez M et al. JC virus load in progressive multifocal leukoencephalopathy: analysis of the correlation between the viral burden in cerebrospinal fluid, patient survival, and the volume of neurological lesions. Clin Infect Dis, 2002;34:1568-75.

4. Hoffmann C, Ernst M, Meyer P, et al. Evolving characteristics of toxoplasmosis in patients infected with HIV-1: clinical course and Toxoplazma gondispecific immune responses. Clin Microbiol Infect, 2007;13:510-5.

5. Karen A. Tuckeret, et al. Neuroimaging in human immunodeficiency virus infection. Journal of Neuroimmunology, 2004;157:153-162.

6. Major EO, Amemiya K, Tornatore CS, Houff SA, Berger JR. Pathogenesis and molecular biology of progressive multifocal leukoencephalopathy, the JC virus-indused demyelinating disease of the human brain. Clin Microbiol Rev, 1992;5:49-73.

7. Manenti G, Di Giuliano F et. al., Case Report: A Case of Primary T-Cell Central Nervous System Lymphoma: MR Imaging and MR Spectroscopy Assessment. Hindawi Publishing Corporation Case Reports in Radiology, 2013, Article ID 916348, 5p.

8. Porter SB, Sande MA. Toxoplasmosis of the central nervous system in AIDS. NEJM 1992;327:1643-8. 